\title{
A simple and novel method to obtain an FSR free silicon ring resonator
}

\author{
Ang $\mathrm{Li}^{\mathrm{a}, \mathrm{b}}$ and Wim Bogaerts ${ }^{\mathrm{a}, \mathrm{b}, \mathrm{c}}$

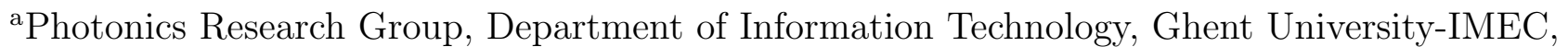 \\ Ghent, Belgium. \\ ${ }^{\mathrm{b}}$ Center for Nano- and Biophotonics (NB-photonics), Ghent University, Belgium. \\ ${ }^{c}$ Luceda Photonics, Dendermonde, Belgium.
}

\begin{abstract}
A simple and novel method to make a silicon ring resonator without the limit of free spectral range (FSR) is presented. For many applications, a ring resonator is desired with an ultra-wide FSR, a high quality factor, a large extinction ratio and low-fabrication complexity. In this paper we propose a novel method to obtain such a ring resonator, which is a single all-silicon microring with a FSR as large as $150 \mathrm{~nm}$ around $1550 \mathrm{~nm}$. It's based on the well-known phenomenon of resonance splitting.
\end{abstract}

Keywords: Microring resonators, Silicon Photonics, Single mode laser cavities, Coupled mode theory, Resonance splitting

\section{INTRODUCTION}

Due to its ultra high $Q$ factor, large extinction ratio (ER) and compact size, ring resonators have become one of the most intensively used optical components for various applications, including optical sensors, WDM filters, laser cavities, optical switches, high-speed modulators ${ }^{1-5}$ etc. There are many platforms to demonstrate ring resonators. Among them, Silicon-On-Insulator has attracted more and more attention in past decades, mainly for its ultra-high index contrast and CMOS processing compatibility. ${ }^{6}$ Thus using SOI gives the possibility to achieve a large-scale and low-cost fabrication of integration photonics.

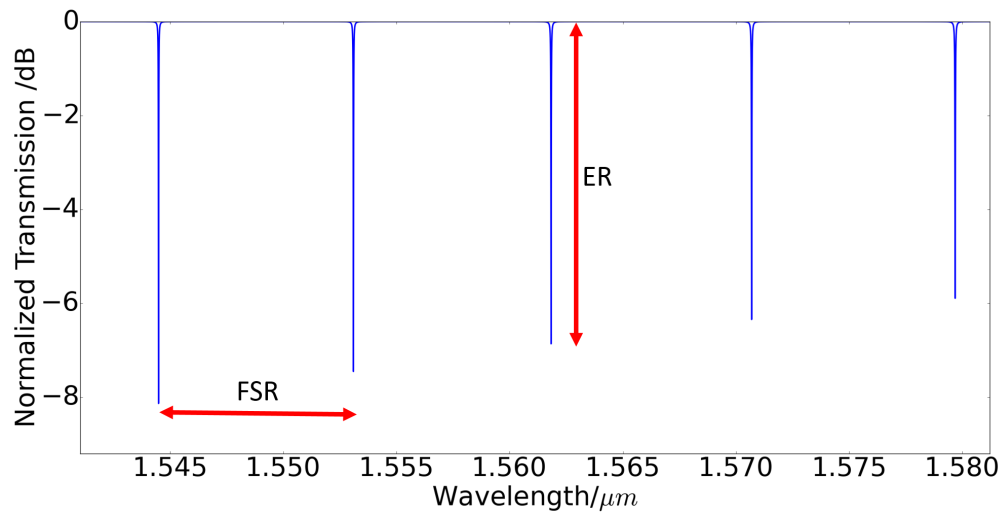

Figure 1. In the output spectrum of an ideal ring resonator, each resonance is a single Lorentzian-shape line.

An ideal ring resonator has a transmission spectrum consisting of a series of Lorentzian-shape resonance lines, as shown in Fig. 1. The optical separation between two adjacent resonances is one of the most important performance indicators of a ring resonator, namely the free spectral range (FSR). Many applications desire the

Further author information: (Send correspondence to Ang Li)

Ang Li: E-mail: ang.li@ugent.be

Wim Bogaerts: E-mail: wim.bogaerts@ugent.be 
ring resonator to have a relatively large FSR as the working range is usually limited to the span of a single FSR. For instance, this corresponds to the sensing range of ring resonator based optical sensors. It would become difficult or impossible to correctly detect the index change if the resonance shift exceeds one FSR. ${ }^{3}$ The same can be said about ring resonator based single mode laser cavities, as the FSR of the ring resonator will limit the tuning range of the laser. ${ }^{5}$ The FSR is dependent on the parameters of a ring resonator as the equation (1) shows:

$$
F S R=\frac{\lambda_{0}^{2}}{n_{g} L}
$$

Here $\lambda_{0}, n_{g}$ and $L$ are the resonance wavelength, the group index and the physical length of the ring waveguide, respectively. Thus in order to get a wide FSR, a relatively short length is needed. This reveals another advantage of the SOI platform for ring resonators: because of the ultra high index contrast, very tight bends and therefore compact rings are possible. ${ }^{7}$ A lot of research has been spent on shortening the total roundtrip length of silicon ring resonators, to obtain a larger FSR. So far the shortest fabricated silicon ring resonator is reported to be $4 \pi \mu m$ long, with a $2 \mu \mathrm{m}$ bend radius. ${ }^{7}$ This generates an FSR just a bit short of $50 \mathrm{~nm}$. The length, however, cannot not be infinitely reduced due to three reasons. Firstly, the tuning mechanism will be difficult to implement. Secondly, it will become difficult or even impossible to obtain the correct coupling coefficient of the directional coupler because of its extremely short coupling length. Finally, short lengths require sharp bend radius. Based on current processing technology, a bend radius smaller than $4 \mu \mathrm{m}$ might already introduce multiple unwanted effects, including extra radiation loss and more backscattering as the mode profile is located closer to the rough outer wall. ${ }^{8,9}$ Potential bend/straight transition loss and reflection would also be induced due to the large mode mismatch between a sharp bend section and a straight waveguide.

Besides directly reducing the ring roundtrip length, there have been many other attempts to increase the FSR of a silicon ring resonator. ${ }^{10-14}$ They either take advantage of the Vernier effect in multiple microrings ${ }^{12,13}$ or introduce intentional reflection inside the ring. ${ }^{10,11,14}$ Both approaches have some drawbacks. For the first one, the design accuracy and fabrication tolerance needs careful attention as the resonance wavelength as well as the bandwidth of each ring need to be perfectly matched, otherwise strong resonance splitting might be induced. Moreover, the tuning scheme will become more complicated to get a continuous shift of the resonance compared to that of a single ring resonator. While the second method introduces CMOS processing incompatibilities to define the backscatterers (e-beam gratings or metal particles), which will negate the advantages of SOI platform. Besides, Bragg grating and metal particles will introduce extra loss mechanisms, i.e. radiation loss and absorption loss. As a consequence, the quality factor and extinction ratio of these ring resonators are relatively poor, as evident in. ${ }^{10}$

Our approach to define a large-FSR ring resonator is based on a single all-silicon ring resonator. Similar with former literature ${ }^{10,11}$ we also widen the FSR by means of internal reflection inside the ring. However, the significant improvement lies in the choice of reflector: an all-silicon loop-MZI based reflector. Thus the whole device is CMOS compatible and no extra loss is introduced in the device. It has a FSR as wide as $150 \mathrm{~nm}$ around $1550 \mathrm{~nm}$. In next section we will explain the principle using temporal coupled mode theory (tCMT) in detail. In the section after that, the design strategy and simulation results will be discussed in detail. Finally comes the conclusion.

\section{PRINCIPLE}

Temporal coupled mode theory (tCMT) has been popularly applied for analyzing ring resonators. ${ }^{15-18}$ In following section we will first introduce tCMT to model an ideal ring resonator and get a good understanding of both tCMT and ring resonators. Subsequently we will use tCMT to explain our method to obtain an ultra wide FSR silicon ring resonator. Finally the schematic of our device will be given.

\subsection{Ideal ring resonator}

First of all, we need to clarify that in tCMT a ring resonator is considered as a lumped oscillator with a timevariant energy stored inside. Here it's the concept of energy that is focused on instead of the electric field.

For an ideal ring resonator, the two circulating modes, namely clockwise (CW) mode and counter-clockwise $(\mathrm{CCW})$ mode, are degenerate and only one of them is active when only one input port is injected with light. Fig. 


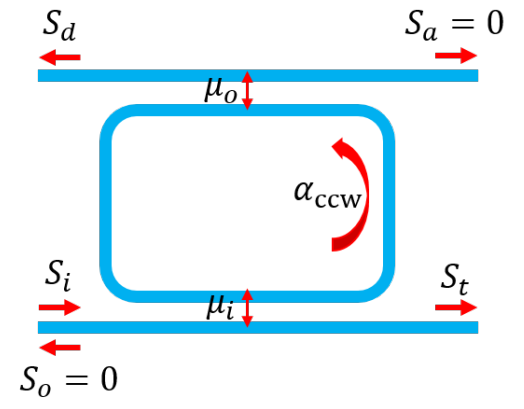

(a)

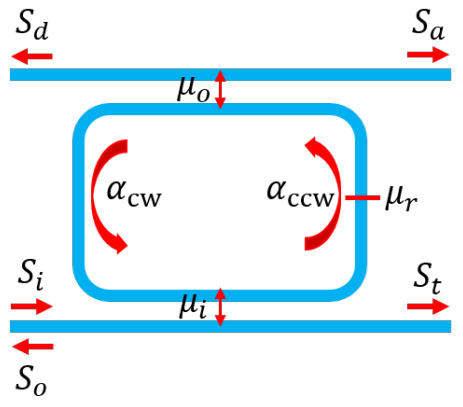

(b)

Figure 2. Schematics of (a) an ideal ring resonator and (b) a ring resonator with internal reflection in tCMT. Ideally, only one circulating mode (for instance, $\alpha_{c c w}$ is active when only one input is injected with light. While due to reflection, both modes are activated simultaneously. $\mu_{x}$ stands for the mutual coupling between two waves.

2 shows the schematics of an ideal ring resonator as well as a ring resonator with internal reflection that couples two circulating modes. Equations (2) and (3) are based on tCMT describing the amplitudes of the circulating mode and transmitted wave at each port:

$$
\begin{gathered}
\frac{d \alpha_{c c w}}{d t}=j \omega_{0} \alpha_{c c w}-\left(\frac{1}{\tau_{l}}+\frac{1}{\tau_{i}}+\frac{1}{\tau_{o}}\right) \alpha_{c c w}-j \mu_{i} S_{i} \\
S_{t}=S_{i}-j \mu_{i} \alpha_{c c w} \quad S_{d}=j \mu_{o} \alpha_{c c w} \quad S_{a}=S_{r}=0
\end{gathered}
$$

Here $\alpha_{c c w}$ refers to the energy amplitude of the mode stored in the ring resonator. It's square $\alpha_{c c w}^{2}$ stands for the total energy of this mode. $S_{x}$ represents the wave amplitude at each port, it's normalized in such a way that its square has the unit of power, quite similar with the concept of field amplitude. Three decay rates $\frac{1}{\tau_{l}}, \frac{1}{\tau_{i}}, \frac{1}{\tau_{o}}$ are due to the intrinsic loss of the ring resonator, coupling to the two side waveguides, respectively. Those factors denoted by $\mu_{x}$ represent the mutual coupling of the couplers. Their physical meanings could be understood by equations (4):

$$
\mu_{i}^{2}=\kappa_{i}^{2} \frac{c}{n_{g} L}=\frac{2}{\tau_{i}} \quad \mu_{o}^{2}=\kappa_{o}^{2}=\frac{2}{\tau_{o}} \frac{c}{n_{g} L} \quad a_{l}^{2} \frac{c}{n_{g} L}=\frac{2}{\tau_{l}}
$$

Here, $c$ is the light speed in vacuum. Factor $\kappa_{x}$ is the common denote for field coupling coefficient of the coupler. $a_{l}$ is an artificial representation of the roundtrip loss of the electric field in the ring waveguide. It's similar to the field coupling coefficient as it could be understood as coupling coefficient to the unguided mode (loss). The mathematical formula for the normalized transmission at the through port can be derived from equations (2) and (3), as shown in equations (5) and (6):

$$
\begin{gathered}
\frac{S_{t}}{S_{i}}=1-\frac{\frac{2}{\tau_{i}}}{\left(\omega-\omega_{0}\right)+\left(\frac{1}{\tau_{l}}+\frac{1}{\tau_{i}}+\frac{1}{\tau_{o}}\right)} \\
\frac{S_{t}^{2}}{S_{i}^{2}}=1-\frac{\frac{2}{\tau_{i}}\left(\frac{2}{\tau_{o}}+\frac{2}{\tau_{l}}\right)}{\left(\omega-\omega_{0}\right)^{2}+\left(\frac{1}{\tau_{l}}+\frac{1}{\tau_{i}}+\frac{1}{\tau_{o}}\right)^{2}}
\end{gathered}
$$

In the ideal case, the output spectrum is a single Lorentzian shape, which corresponds well with the theory. One of the most important performance indicators of ring resonator is its extinction ratio (ER). It's this parameter that we are going to play with to make the FSR of the our device very wide. ER is directly determined by the transmission at the resonance frequency $\omega=\omega_{0}$. For an ideal ring, it is given in equation (7):

$$
P_{0}=1-\frac{\frac{2}{\tau_{i}}\left(\frac{2}{\tau_{o}}+\frac{2}{\tau_{l}}\right)}{\left(\frac{1}{\tau_{l}}+\frac{1}{\tau_{i}}+\frac{1}{\tau_{o}}\right)^{2}}
$$


When $\frac{1}{\tau_{i}}=\frac{1}{\tau_{l}}+\frac{1}{\tau_{o}}, P_{0}$ becomes 0 , which gives the largest ER. That is the so-called critical coupling of ring resonator. Physically speaking, when the light coupled in from input equals the light leaking out of ghe ring resonator (loss + coupled out to the output), we get the largest ER.

\subsection{Ring resonator with internal reflection}

After building a tCMT model for an ideal ring resonator, we will now apply tCMT again to analyze a ring resonator with internal reflection. This analysis will reveal how the ER of one resonance could be strongly modified by the reflectivity inside the ring. Based on this phenomenon we are able to make a ring resonator with ultra-wide FSR.

The most essential difference with an ideal ring resonator is that due to internal reflection, the two circulating modes $\mathrm{CW}$ and $\mathrm{CCW}$ are coupled with each other, thus simultaneously activated by only one input port. The modes in the resonator will be changed from a single mode state to a more complicated coupled mode state. Still based on tCMT, equations for the two circulating modes are given in equations (8) and (9):

$$
\begin{gathered}
\frac{d \alpha_{c c w}}{d t}=j \omega_{0} \alpha_{c c w}-\left(\frac{1}{\tau_{l}}+\frac{1}{\tau_{i}}+\frac{1}{\tau_{o}}\right) \alpha_{c c w}-j \mu_{r} \alpha_{c w}-j \mu_{i} S_{i} \\
\frac{d \alpha_{c w}}{d t}=j \omega_{0} \alpha_{c w}-\left(\frac{1}{\tau_{l}}+\frac{1}{\tau_{i}}+\frac{1}{\tau_{o}}\right) \alpha_{c w}-j \mu_{i} S_{i}
\end{gathered}
$$

Here, a new term $\mu_{r}$ appears. Similar to $\mu_{o}, \mu_{i}$ this stands for the mutual coupling of the reflection that couples the two modes. Here the coupling of these two modes, namely reflection in our case, is considered to be conservative instead of dissipative. Its physical meaning is indicated in equation (10):

$$
\mu_{r}^{2}=r^{2} \frac{c}{n_{g} L}
$$

Field reflectivity is denoted by $r$. Equations for transmitted wave amplitude at each port is given by:

$$
S_{t}=S_{i}-j \mu_{i} \alpha_{c c w} \quad S_{d}=S_{i}-j \mu_{o} \alpha_{c c w} \quad S_{a}=-j \mu_{o} \alpha_{c w} \quad S_{r}=-j \mu_{i} \alpha_{c w}
$$

The equations for $S_{t}$ and $S_{d}$ remain the same (but the $\alpha_{c c w}$ has changed), while $S_{a}$ and $S_{r}$ are not zero any more. Physically speaking, due to the activation of mode $\alpha_{c w}$, there is undesired light passing to add port and reflected to in port. Solving equations (8), (9) and (11) we get a modified formula for the normalized transmission at through port:

$$
\begin{aligned}
\frac{S_{t}}{S_{i}} & =1-\frac{2}{\tau_{i}} \frac{j\left(\omega-\omega_{0}\right)+\left(\frac{1}{\tau_{l}}+\frac{1}{\tau_{i}}+\frac{1}{\tau_{o}}\right)}{\left[j\left(\omega-\omega_{0}\right)+\left(\frac{1}{\tau_{l}}+\frac{1}{\tau_{i}}+\frac{1}{\tau_{o}}\right)\right]^{2}+\mu_{r}^{2}} \\
& =1-\frac{2}{\tau_{i}}\left(\frac{0.5}{j\left(\omega-\omega_{1}\right)+\left(\frac{1}{\tau_{l}}+\frac{1}{\tau_{i}}+\frac{1}{\tau_{o}}\right)}+\frac{0.5}{j\left(\omega-\omega_{2}\right)+\left(\frac{1}{\tau_{l}}+\frac{1}{\tau_{i}}+\frac{1}{\tau_{o}}\right)}\right)
\end{aligned}
$$

Obviously, instead of one Lorentzian-shape resonance, now the output spectrum contains two Lorentzian-shape resonances with their own central frequencies, $\omega_{1}=\omega_{0}+\left|\mu_{r}\right|$ and $\omega_{2}=\omega_{0}-\left|\mu_{r}\right|$. Subsequently the transmission at resonance frequency is modified to equation (13):

$$
P_{0}=\left(\frac{\frac{2}{\tau_{l}}+\frac{2}{\tau_{o}}}{\frac{2}{\tau_{l}}+\frac{2}{\tau_{o}}+\frac{2}{\tau_{i}}}\right)^{2}+\frac{\left(\frac{2}{\tau_{i}}\right)^{2}-2 \frac{2}{\tau_{i}}\left(\frac{2}{\tau_{l}}+\frac{2}{\tau_{o}}\right)}{\left(\frac{2}{\tau_{l}}+\frac{2}{\tau_{o}}+\frac{2}{\tau_{i}}\right)^{2}+16\left|\mu_{r}^{2}\right|}
$$

To get a better understanding of how these physical parameters, especially the reflectivity, would influence the transmission and ER, we replace factors $\tau_{x}$ and $\mu_{r}$ in equation (13) with coupling coefficients $\kappa_{x}$ and reflectivity $r$ through equations (4) and (10) and we get:

$$
P_{0}=\left(\frac{\kappa_{0}^{2}+a_{l}^{2}}{\kappa_{i}^{2}+\kappa_{o}^{2}+a_{l}^{2}}\right)^{2}+\frac{\left(\kappa_{i}^{2}\right)^{2}-2 \kappa_{i}^{2}\left(\frac{2}{\tau_{l}}+\frac{2}{\tau_{o}}\right)}{\left(\frac{2}{\tau_{l}}+\frac{2}{\tau_{o}}+\frac{2}{\tau_{i}}\right)^{2}+16\left|\mu_{r}^{2}\right|}
$$




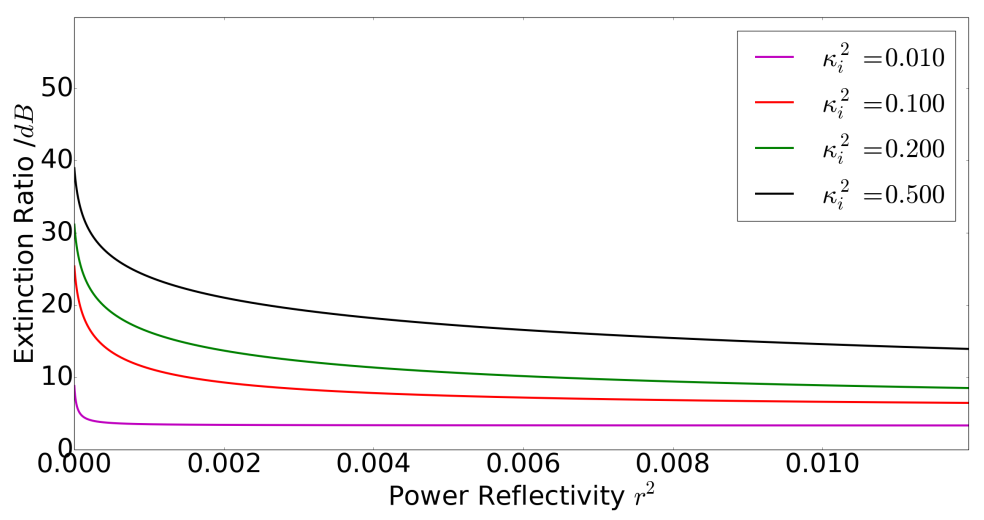

Figure 3. The extinction ratio(ER) of a resonance drops dramatically with increasing reflectivity inside the ring.

When $r=0$, equation (14) becomes identical with equation (7). Now it's too complicated to directly analyze. In figure 3 it's shown how the ER of one resonance changes with reflectivity $r$, when $\kappa_{o}=\kappa_{i}$, which is often the case and easy to guarantee. Generally speaking, ER drops dramatically with increasing reflectivity $r$. This is the basic principle for our device. If we could design a reflector, whose reflection spectrum is strongly wavelength dependent, specifically speaking, only one wavelength sees zero reflection while all other wavelengths suffer strong reflection. Then for the ring resonator's spectrum, we will see that only one resonance that matches with the reflector's zero-reflection wavelength has a very large ER while the rest resonances have very small ER. Under this circumstance, we could consider this ring resonator as a single-mode resonator, whose FSR depends on the FSR of the reflector.

The reflector we propose is a Mach-Zehnder-Interfereometer ended with a Sagnac mirror. In next section we will give the design strategy of the device and its simulation results using a circuit simulator-Caphe. ${ }^{19}$

\subsection{Schematic}
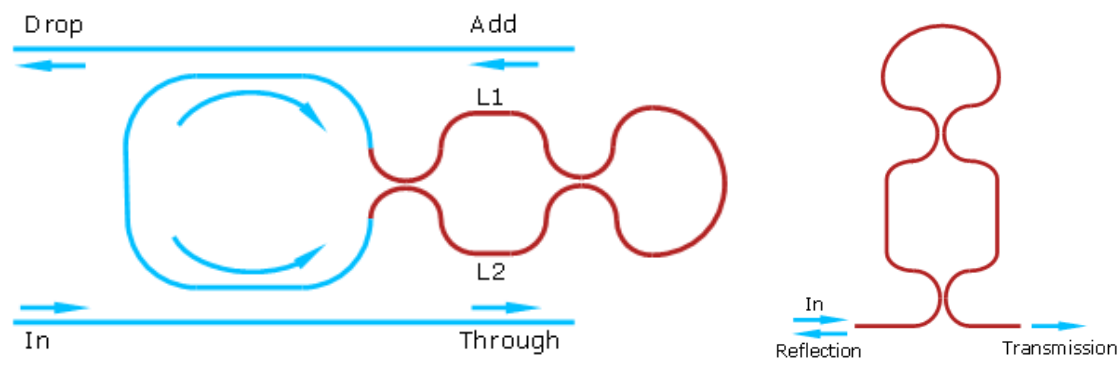

Figure 4. Schematic of our device (left) and the MZI based reflector (right).

The schematic of the whole device is given in figure 4. It could be simply divided into two parts, a ring resonator and a reflector. The reflector itself consists of two parts: a tunable MZI and a Sagnac mirror connected with its output.

\section{DESIGN AND SIMULATION}

In this section, we will first discuss about the design strategy as well as the key parameters of our device. Then the simulation results using our circuit simulator-Caphe will be given in detail.

As mentioned in last section, if we manage to make a reflector, in whose spectrum only one wavelength has zero reflection while the rest suffer strong reflection, then in terms of the ring spectrum, only one resonance has 
a large $E R_{0}$ with all the rest being suppressed to a very small $E R_{1}$. And we define a new important parameter for our device, the side mode suppression ratio (SMSR) as $S M S R=E R_{0}-E R_{1}$. If SMSR is large enough, we may consider the ring as a single resonance ring, whose FSR depends on the FSR of the reflector instead of the ring length.

The design principle of the reflector is quite similar with that of a normal MZI. The key design freedom is the length difference $\Delta L$ between its two arms $L_{1}$ and $L_{2}$. The absolute length of these two arms depends on the applications of this ring. For instance, for a ring biosensors, a relatively longer length is useful to expose a long waveguide to bio medium. On the other hand, for WDM filters the length should be designed to be as short as possible to minimize the footprint, the loss and stochastic backscattering. ${ }^{15}$ The zero-reflection wavelength point is:

$$
\frac{2 \pi \Delta L n_{e f f}}{\lambda}=m \pi
$$

where $m$ is the interference order, similar to that of an MZI. The reflection spectra for different interference orders $m$ or $\Delta L$ generated by Caphe are plotted in figure 5 . The simulated device contains two 50/50 directional couplers with a wavelength-dependent model extracted from a commercial FDTD simulator-Lumerical and the zero-reflection wavelength is set to be $1540 \mathrm{~nm}$. The FSR of the reflector is as large as $150 \mathrm{~nm}$. The influence of $\Delta L$ is clearly demonstrated in this figure. A longer $\delta L$ will lead to a sharper slope, which means a higher efficiency or a larger SMSR.

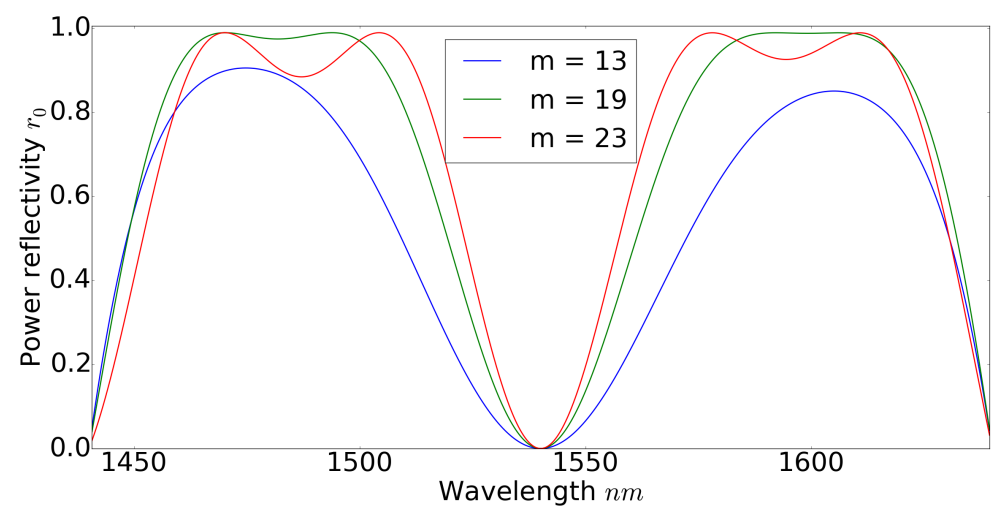

Figure 5. Different reflection spectra simulated with Caphe. Each color corresponds with a interference order $m$ or $\Delta L$. Longer $\Delta L$ can generate a sharper slope, which means a larger SMSR.
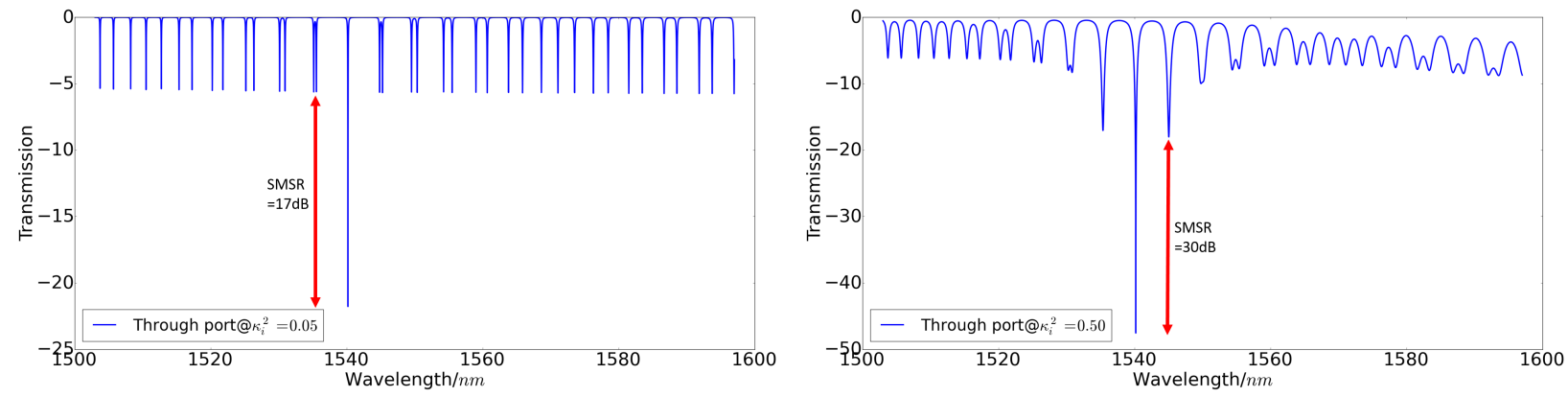

Figure 6. Simulated 'through' port of our devices at two different configurations, $\kappa_{o}^{2}=\kappa_{i}^{2}=0.05,0.5$, respectively.

The simulation result of the complete device is given in figure $6 . m$ is chosen to be 23 , and the ring resonator has a power coupling coefficient of $\kappa_{o}^{2}=\kappa_{i}^{2}=0.05$ and $\kappa_{o}^{2}=\kappa_{i}^{2}=0.5$ respectively, to show that our device could work under a very large range of coupling coefficient compared with a normal ring resonator that generally requires a ring to be configured at its critical coupling point. The SMSR could be as large as $30 \mathrm{~dB}$ at $\kappa_{i}^{2}=0.5$. 


\section{CONCLUSION}

In this paper we present a novel method to design an ultra-wide FSR silicon ring resonator. It's a single all-silicon ring resonator with a FSR as large as $150 \mathrm{~nm}$. The potential applications include single mode laser, integrated sensors, WDM filters etc.

\section{REFERENCES}

[1] Emelett, S. J. and Soref, R., "Design and simulation of silicon microring optical routing switches," Journal of lightwave technology 23(4), 1800 (2005).

[2] Malacarne, A., Gambini, F., Faralli, S., Klamkin, J., and Poti, L., "High-speed silicon electro-optic microring modulator for optical interconnects," Photonics Technology Letters, IEEE 26, 1042-1044 (May 2014).

[3] Hoste, J.-W., Werquin, S., Claes, T., and Bienstman, P., "Conformational analysis of proteins with a dual polarisation silicon microring," Optics express 22(3), 2807-2820 (2014).

[4] Little, B. E., Chu, S. T., Haus, H. A., Foresi, J., and Laine, J.-P., "Microring resonator channel dropping filters," Lightwave Technology, Journal of 15(6), 998-1005 (1997).

[5] Lee, J. H., Shubin, I., Yao, J., Bickford, J., Luo, Y., Lin, S., Djordjevic, S. S., Thacker, H. D., Cunningham, J. E., Raj, K., et al., "High power and widely tunable si hybrid external-cavity laser for power efficient si photonics wdm links," Optics express 22(7), 7678-7685 (2014).

[6] Bogaerts, W., Dumon, P., Van Thourhout, D., Taillaert, D., Jaenen, P., Wouters, J., Beckx, S., Wiaux, V., and Baets, R. G., "Compact wavelength-selective functions in silicon-on-insulator photonic wires," Selected Topics in Quantum Electronics, IEEE Journal of 12(6), 1394-1401 (2006).

[7] Nawrocka, M. S., Liu, T., Wang, X., and Panepucci, R. R., "Tunable silicon microring resonator with wide free spectral range," Applied physics letters 89(7), 71110-71110 (2006).

[8] Selvaraja, S. K., Bogaerts, W., and Van Thourhout, D., "Loss reduction in silicon nanophotonic waveguide micro-bends through etch profile improvement," Optics Communications 284(8), 2141-2144 (2011).

[9] Shen, H., Fan, L., Wang, J., Wirth, J. C., and Qi, M., "A taper to reduce the straight-to-bend transition loss in compact silicon waveguides," Photonics Technology Letters, IEEE 22(15), 1174-1176 (2010).

[10] Urbonas, D., Balčytis, A., Gabalis, M., Vaškevičius, K., Naujokaitè, G., Juodkazis, S., and Petruškevičius, R., "Ultra-wide free spectral range, enhanced sensitivity, and removed mode splitting soi optical ring resonator with dispersive metal nanodisks," Opt. Lett. 40, 2977-2980 (Jul 2015).

[11] Huang, Q., Ma, K., and He, S., "Experimental demonstration of single mode- splitting in microring with bragg gratings," Photonics Technology Letters, IEEE 27, 1402-1405 (July 2015).

[12] Oda, K., Takato, N., and Toba, H., "A wide-fsr waveguide double-ring resonator for optical fdm transmission systems," Lightwave Technology, Journal of 9(6), 728-736 (1991).

[13] Geuzebroek, D., Klein, E., Kelderman, H., Tan, F., Klunder, D., and Driessen, A., "Thermally tuneable, wide fsr switch based on micro-ring resonators," (2002).

[14] Boeck, R., Caverley, M., Chrostowski, L., and Jaeger, N. A. F., "Grating-assisted silicon-on-insulator racetrack resonator reflector," Opt. Express 23, 25509-25522 (Oct 2015).

[15] Li, A., Vaerenbergh, T. V., peter de heyn, Xing, Y., Bienstman, P., and Bogaerts, W., "Experimentally demonstrate the origin for asymmetric resonance splitting and contributions from couplers to backscattering in soi microrings," in [Advanced Photonics 2015], Advanced Photonics 2015, IM2B.6, Optical Society of America (2015).

[16] Haus, H., [Waves and fields in optoelectronics], Prentice-Hall Series in Solid State Physical Electronics, Prentice Hall, Incorporated (1984).

[17] Little, B., Chu, S., Haus, H., Foresi, J., and Laine, J.-P., "Microring resonator channel dropping filters," Journal of Lightwave Technology 15, 998-1005 (June 1997).

[18] Popović, M. A., Theory and Design of High-Index-Contrast Microphotonic Circuits, PhD thesis (2008).

[19] Fiers, M., Vaerenbergh, T. V., Caluwaerts, K., Ginste, D. V., Schrauwen, B., Dambre, J., and Bienstman, P., "Time-domain and frequency-domain modeling of nonlinear optical components at the circuit-level using a node-based approach," J. Opt. Soc. Am. B 29, 896-900 (May 2012). 